\title{
Ein elementares Werk zu den Numeralien des Udmurtischen
}

О.Б. Стрелкова: Имена числительные удмуртского языка. История и типология: монография [Die Numeralien der udmurtischen Sprache. Geschichte und Typologie: Monografie]. Ижевск 2013. $238 \mathrm{~S}$.

Olga Strelkovas Monografie ist eine fundierte Arbeit über die Numeralien im Udmurtischen. Sie behandelt das System der Zahlwörter ebenso wie ihre Flexion, Ableitung und Etymologie. Das vorliegende Werk vertritt den gleichen Typ wie einige andere in letzter Zeit verfasste Abhandlungen über das Udmurtische, die offenkundig den Zweck verfolgen, eine grundlegende deskriptive Darstellung der Sprache $\mathrm{zu}$ liefern und sie so zu normieren, dass sie den heutigen Anforderungen des Sprachgebrauchs gerecht wird.

Das Buch vermittelt ein klares Bild vom Charakter der udmurtischen Zahlwörter im Sprachsystem. Sowohl die Kardinal- als auch die Ordnungszahlen gehören $\mathrm{zu}$ den Wörtern, bei denen die Verwendung des Determinativsuffixes möglich ist. Interessant ist in dieser Hinsicht auch, dass im
Udmurtischen die Possessivsuffixe aller Personen bei den Zahlwörtern ebenfalls verwendet werden können. (S. 13-14, 17, 74, 121.) Die Verbindung zwischen Possessivund Determinativsuffix sowie die Grenzziehung zwischen ihnen ist ein weiterhin aktuelles Problem der udmurtischen Sprachforschung.

Strelkova muss auch zu eher trivial erscheinenden Fragen Stellung nehmen; sie muss z. B. begründen, weshalb Wörter, die eine unbestimmte Menge bezeichnen, keine Zahlwörter sind. Diese wurden nämlich in einigen früheren udmurtischen Grammatiken als $\mathrm{Nu}-$ meralien klassifiziert. (S. 126-135.) Hier beruft sich die Verfasserin vor allem darauf, dass Wörter, die eine unbestimmte Menge bezeichnen, nicht zum Wesen des Zahlwortsystems passen, auch wenn sie semantisch als nahestehend betrachtet werden können.

Der Plural des Zahlwortes bedeutet oft Approximativität. Im Udmurtischen ist dies eine von vielen Mitteln, Approximativität auszudrücken. Andere gebräuchliche Mittel sind Postpositionen mit der Bedeutung, ungefähr' sowie das spezielle, eine annähernde Menge 
bezeichnende Suffix -o. Zudem kann man mehrere Mittel kombinieren. Strelkova stellt die verschiedenen Mittel und ihre Kombinationen übersichtlich dar. Dabei erhebt sich die Frage, warum die Sprache über so viele unterschiedliche, synonyme Ausdrucksmittel verfügt. Auch die Kurzform oz des Zahlworts, eins' hat im Udmurtischen im Allgemeinen die Bedeutung ,ungefähr'. Eine ähnliche Verwendung des Zahlworts eins ist auch im Mari sowie in den türkischen und iranischen Sprachen verbreitet. (S. 81-82.) Auch an einigen anderen Stellen wird auf türkischen und iranischen Einfluss hingewiesen; dies ergibt ein gutes Gesamtbild von der Richtung vieler sprachlicher Einflüsse, was für Erforscher des Udmurtischen von Nutzen ist, deren Arbeit einen historischen oder etymologischen Aspekt einschließt.

Dargestellt wird auch, welche Substantive mit Ableitungssuffix von den Zahlwörtern gebildet werden können (S. 136-139). Mit diesen Ableitungssuffixen wurden in letzter Zeit im Udmurtischen neue wissenschaftliche Termini gebildet. Im Udmurtischen sind die Zahlwörter relativ vital, d.h. auch in der gesprochenen Sprache werden eigensprachliche Zahlwörter erheblich häufiger verwendet als z. B. im nahe verwandten Komipermjakischen, wo man weitaus häufiger zu russischen Zahlwörtern greift. Ein ungenutztes Potenzial findet sich im Udmurtischen auch bei den denumeralen Ableitungen. Im Prinzip könnte man mit dem Ableitungssuffix $-\varkappa \mathcal{u}$ beispielsweise von allen üblichen Zeugnisnoten Substantive bilden, die eine Person bezeichnen: куиньчи ,Studierender mit der Note 3 , ньыльчи, Studierender mit der Note 4, витьчu ,Studierender mit der Note 5 '. Stattdessen werden die entsprechenden russischen Wörter троечник, хороиист, отличник verwendet. Nur der eigensprachliche Ausdruck кькчи, Studierender mit der Note $2^{c}$ ist heute tatsächlich in Gebrauch. (S. 139.) Die Zahlwörter sind einer der speziellen Segmente der Sprache, die bei starkem Sprachkontakt und Zweisprachigkeit oft leiden, wenn höhere Bildung nur in einer Sprache zugänglich ist. Auch deshalb sind sowohl Strelkovas Thema generell als auch die Berücksichtigung der Rolle der Zahlwörter bei der Entwicklung der Sprache ausgesprochen aktuell und wichtig.

Mit großer Sorgfalt behandelt Strelkova auch das Suffix der Bezeichnungen für Bruchzahlen, das in der heutigen Sprache wie ein spezielles Bruchzahlsuffix erscheint, im Gegensatz zu vielen anderen uralischen Sprachen, wo die Bruchzahl mit Hilfe der Ordnungszahl ausgedrückt wird. Bei genauerer 
Betrachtung zeigt sich, dass das betreffende udmurtische Suffix eine Entlehnung aus dem Tschuwaschischen ist und ursprünglich die Ordnungszahl markierte. (S. 96.)

Das Werk befasst sich auch mit der Frage der Relikte des Duals in den uralischen Sprachen. In einigen uralischen Sprachen gibt es weiterhin einen numerischen Dual, wie in der uralischen Ursprache. Im Udmurtischen gibt es keinen Dual. $\mathrm{Zu}$ diesem Themenkreis gehört die Tatsache, dass man im Udmurtischen weiterhin bei ihrem Wesen nach paarweise auftretenden Designaten im Allgemeinen den Singular für das Paar verwendet. So war es offenbar auch in der uralischen Ursprache. Man vermutet, dass solche Paarwörter der Grund für die Entstehung des Duals z. B. in der indogermanischen Sprachfamilie waren. (S. 156-157.) Nicht wirklich begründet ist jedoch Strelkovas These, wonach die Tatsache, dass in einigen heutigen Sprachen, wie im Ungarischen und Udmurtischen, der eine Bestandteil paarweise auftretender Gegenstände mit dem Wort ,halb' bezeichnet wird, während der Singular auf das Paar verweist, ein Relikt des Duals sei (S. 158): Ist die Wahrnehmung paarweiser Begriffe als Singular nicht schon an sich ein hinreichender Grund für die Verwendung des Wortes ,halb` in diesem Kontext?
Strelkova erwähnt ein interessantes Phänomen im Udmurtischen: Die Verwendung des Wortes пал in der Bedeutung ,halb / einer, der eine der beiden Teile eines Paares' hat sich auch auf Wörter erweitert, die nicht von Natur aus paarweise Dinge bezeichnen. So haben z. B. die Ausdrücke пал кыл („halbes Wort“) und пал кисььри („halbe Falte") die Bedeutung, ein Wort" und ,eine Falte. (S. 159.) Solche Erscheinungen müssen z. B. bei Übersetzungen von einer Sprache in die andere beachtet werden. Auch im Finnischen könnte man im Prinzip entsprechende Ausdrücke bilden: sanapuoli, ryppypuoli, aber eine solche Verwendung dürfte im Finnischen nur möglich sein, wenn es sich um konkret paarweise auftretende Designate handelt.

Der Numerus des Substantivs in Verbindung mit einem Zahlwort als nähere Bestimmung ist im Udmurtischen insofern eine interessante Frage, als sowohl der Singular wie auch der Plural möglich ist. Strelkova stützt sich bei ihrer Analyse auch auf quantitative Erhebungen aus schriftlichen Texten. Aufgrund der gegenwärtigen Erkenntnisse sind drei Umstände festzustellen, die die Wahl des Numerus beeinflussen: 1. Früher war der Singular häufiger als heute. Die Verbreitung des Pluralgebrauchs geschah offenbar unter dem Einfluss des Russischen. 2. 
Wenn zwischen dem Zahlwort und dem Bezugswort noch eine zweite Bestimmung des Substantivs steht, ist der Plural wahrscheinlicher. Eine solche Konstruktion ist den Substantivphrasen vergleichbar, die kein Zahlwort enthalten und bei denen folglich immer das Pluralkennzeichen verwendet wird, wenn es sich um einen Plural handelt. 3. Ist das Bezugswort eine Maßeinheit, ein sog. Numerativ, wird der Singular verwendet, in allen anderen Fällen ist auch der Plural möglich. Auch an dieser Stelle verweist Strelkova auf die iranischen Sprachen, in denen die Numerative die gleiche Eigenschaft besitzen. (S. 177-187.) Der Numerus des Substantivs in Verbindung mit einem Zahlwort als Bestimmung ist eine Grundfrage des Sprachgebrauchs, die bisher nicht in dieser Deutlichkeit beschrieben wurde.

Regeln und Praxis der Zusammen- und Getrenntschreibung waren im Udmurtischen teils unklar, und dieses Thema hat bisher wenig Beachtung gefunden. In Übereinstimmung mit anderen heutigen Udmurtologen empfiehlt Strelkova, Adjektive, die ein Zahlwort enthalten, wie куиньаресьем, dreijährig‘, zusammenzuschreiben, da andernfalls Interpretationsunklarheiten entstehen können. (S. 14, 176.)

Auf eine sehr interessante Erscheinung weist Strelkova auf
S. 122-123 hin: Die häufiger werdende Verwendung von Kardinalzahlen anstelle von Ordnungszahlen z. B. bei Datumsangaben sowohl in der gesprochenen als auch in der geschriebenen Sprache, entgegen den Regeln der Grammatik. Zur Erklärung dieses Phänomens weist sie vorsichtig darauf hin, dass das Datum häufig mit arabischen Ziffern geschrieben wird, bei denen nicht unmittelbar zu sehen ist, dass es sich um Ordnungszahlen handelt. Kann die schriftliche Form die Sprache so stark beeinflussen? Das erscheint nicht unbedingt glaubhaft. Sonst müsste man ja annehmen, dass man z. B. im Finnischen das Datum 12.6.200o als kaksitoista kuusi kaksituhatta,zwölf sechs zweitausend' (pro kahdestoista / kahdentenatoista kuudetta kaksituhatta ,zwölfter / am zwölften sechsten zweitausend') lesen würde. Die Kardinalzahl begegnet in diesem Kontext schon von alters her sowohl in der Literatur als auch in den südlichen Dialekten, wie Strelkovas Beispiele zeigen. Es geht hier eher darum, dass die Regel der Grammatik zu streng ist. Die Verwendung der Ordnungszahl beim Datum ist keineswegs ein universales Muss. Im Rumänischen z. B. wird die Kardinalzahl verwendet.

Im Jahr 2009 legte Strelkova ihre Kandidatendissertation über die udmurtischen Zahlwörter vor. 
Die hier besprochene Monografie Morphologie lehrt, hat auch bei der ist auf der Grundlage dieser Ar- Abfassung eines umfangreichen beit entstanden. Sie wird ihrem akademischen Lehrbuchs über Ziel bestens gerecht: Grundwissen Morphologie sowie an der Erstelüber die Numeralien zu vermit- lung von Lehrbüchern des Udmurteln. Das Buch bietet ein zuverläs- tischen für Ausländer und andere siges Fundament, auf das sich alle Sprachunkundige mitgewirkt. Man Wissenschaftler stützen können, wird von ihr auch künftig Beiträge deren Untersuchungen die Nume- auf dem Gebiet der udmurtischen ralien des Udmurtischen tangieren. Sprache erwarten dürfen.

Strelkova, die an der Staatlichen Universität von Udmurtien u.a. Esa-Jussi Salminen 